\title{
A Novel Method to Solve a Class of Non-local Diffusion Optimal Control Problems by using Bernstein Polynomials
}

\author{
Ali Ketabdari \\ Department of Applied Mathematics, Faculty of Mathematical Sciences, Ferdowsi University of Mashhad, \\ Mashhad, Iran \\ E-mail: aliketabdari@mail.um.ac.ir
}

Mohammad Hadi Farahi

Department of Applied Mathematics, Faculty of Mathematical Sciences, Ferdowsi University of Mashhad, Mashhad, Iran \& The Center of Excellence on Modelling and Control Systems (CEMCS), Mashhad, Iran

E-mail: Farahi@math.um.ac.ir

Received: 25 June 2019; Accepted: 22 November 2019; Published: 08 June 2020

\begin{abstract}
The aim of this paper is solving optimal control problems governed by non-local diffusion equations via a mesh-less method. The diffusion equation and in particular, the heat conduction equation is essential in sciences. This equation appears in many fields, such as engineering, electrostatic, and mathematics. For solving the mentioned optimal control problems, the method is established upon expanding of variables by the basis of Bezier functions. We apply, for the first time, the Bernstein approximation in solving an optimal control problem governed by the diffusion equation. A direct algorithm is given for solving this problem. Bernstein polynomials expand the trajectories and control functions with unknown control points. Then the optimal control problem is converted to a mathematical programming problem. By solving the mathematical programming problem, the approximated solution of trajectories and control are driven. The convergence of the method in approximating of the optimal control problem is proved. Some numerical examples for demonstrating the effectiveness of the method are included.
\end{abstract}

Index Terms - Optimal control, diffusion equation, Bezier function, Bernstein polynomial.

\section{INTRODUCTION}

Heat conduction equation is a famous partial differential equation in sciences. The classical parabolic heat conduction equation is shown as follows:

$$
\frac{\partial w}{\partial t}+\lambda \Delta w=h
$$

where $w(x, t)$ shows the temperature in the position $x$ and time $t, \lambda$ is a constant coefficient, $h(x, t)$ is a given continuous and well-behaved function, and $\Delta$ denotes the Laplace operator. The study concerning the heat conduction equation is returned to Fourier's studies about heat conduction. In the last decades, the investigation of the unsteady heat conduction equation is increased. Initially, Morse and Feshbach [1] and then Maxwell [2] formulated some of the unsteady heat conduction equations. In 1959, Carslaw and Jaeger [3] and in 1969, Rotem and Neilson [4] considered the repercussion of heat conduction equations. In 1971, Tamir and Taitel improved work's Rotem; see [5]. In 1972, Taitel presented a solution for a thing with a thin layer by parabolic, hyperbolic, and discrete heat conduction equations (see[6]). By using his work and other scientists, the application of heat conduction equation in the analysis of phenomena and physical processes such as industrial process, long cylinder, and plan wall was incremented. In the recent decade, wise methods for solving this problem have increased. Pavlov and Kudoyarova [7] solved this problem with the Spline method. Al-Khaled [8] used the Fourier regularization method. In many books and articles for solving the heat conduction equation, the finite element method was used (see[9,10,11]); many authors solved this problem by the Meshless method (see[12,13] and references therein).

In recent years, Bezier functions have attracted the attention of researchers. These functions are suitable tools for solving partial differential equations (PDEs) as a meshless method, and also these tools have excellent properties such as a straightforward definition, quick in determination and execution in the computer, continuity property interpolation end-points and symmetry property.

Bezier functions were applied for solving Fredholm integral equations (see[14,15,16]), Volterra integral equations [17], and Volterra-Fredholm-Hammerstein integral equations [18]. Zheng et al. [19] and Safaie and 
Farahi [20] introduced the Bernstein-Bezier form for solving ordinary differential equations and they used an objective function based on the Bezier control points. In this paper, we consider optimal control problems governed by the nonlocal diffusion equation using Bernstein polynomials. To obtain the optimal pair of trajectory and control functions, we use the operational matrices of derivative and integral and then solve the optimal control problem by converting PDE constraints to an algebraic system.

The remainder of this paper is planned as follows: Section 2 introduces some essential properties of Bernstein and Bezier functions. In Section 3, we consider the one-dimensional diffusion equation with initial and boundary conditions and then, by using Bezier function, convert this one-dimensional diffusion equation to a mathematical optimization problem. In Section 4, we convert this problem to a quadratic programming problem with linear constraints and also remind four issues for solving the problem. In Section 5, we give the convergence analysis of the present method. In Section 6, three numerical examples are solved, which show the efficiency and reliability of our method. A conclusion is given in section 7 .

\section{PRELIMINARIES}

Bezier curves have more interesting properties than the cubic or B-splines. For example, in the cubic spline, if we change only two interpolating points, then we need to redetermine the cubic spline function, but in Bezier functions, one can find a new Bezier function from the old one. Bezier functions are used in computer-aided design (CAD) (see[21]), computer graphics to draw shapes, solving the various control problems such as switching control systems (see[22]), and many different applications (see[23,24,25,26,27,28,29]).

This section consists of some basic definitions and properties of Bezier functions.

Definition 2.1 The Bernstein polynomials of degree $n$ over the interval $[0,1]$ are defined as follows:

$$
B_{i}^{n}(t)=\left(\begin{array}{c}
n \\
i
\end{array}\right) t^{i}(1-t)^{n-i}
$$

for $i=0,1,2, \ldots, n$ where $\left(\begin{array}{c}n \\ i\end{array}\right)=\frac{n !}{i !(n-i) !}$.

If one uses the binomial expansion for $(1-t)^{n-i}$, then

$$
B_{i}^{n}(t)=\sum_{k=0}^{n-i}\left(\begin{array}{c}
n-i \\
k
\end{array}\right)\left(\begin{array}{l}
n \\
i
\end{array}\right)(-1)^{k} t^{i+k}
$$

Definition 2.2 Define a Bernstein vector $\varphi_{n}(t)$ as

$$
\varphi_{n}(t)=\left[\begin{array}{llll}
B_{0}^{n}(t) & B_{1}^{n}(t) & \cdots & B_{n}^{n}(t)
\end{array}\right]^{T} ;
$$

then the Bezier polynomial of degree $n$ over the interval $[0,1]$ is defined as follows:

$$
P_{n}(t)=C \varphi_{n}(t)
$$

where

$$
C=\left[\begin{array}{llll}
c_{0} & c_{1} & \cdots & c_{n}
\end{array}\right]
$$

is the vector of constant coefficients that we recall its entry as control points. Thus (3) and (4) implies that

$$
P_{n}(t)=\sum_{i=0}^{n} c_{i} B_{i}^{n}(t)
$$

Lemma 2.3 By using (2), we define $\varphi_{n}(t)=\psi_{n} T_{n}(t)$ where $T_{n}(t)=\left[\begin{array}{llll}1 & t & \cdots & t^{n}\end{array}\right]^{T}$ and $\psi_{n}=\Psi^{n+1}$ is an $(n+1) \times(n+1)$ upper triangular matrix that can be expressed by

$$
\Psi_{i+1, j+1}^{n+1}=\left\{\begin{array}{cc}
\frac{(-1)^{j-i} n !}{(n-j) !(j-i) ! i !}, & i \leq j \\
0, & i>j
\end{array}\right.
$$

for $i, j=0,1, \ldots, n$.

Proof. See [30].

Lemma 2.4 Suppose that $H=L^{2}[0,1]$ and that $\left\{B_{0}^{n}(t), B_{1}^{n}(t), \ldots, B_{n}^{n}(t)\right\} \subset H \quad, \quad$ and $\quad$ let $Y=\operatorname{Span}\left\{B_{0}^{n}(t), B_{1}^{n}(t), \ldots, B_{n}^{n}(t)\right\} . \quad Y \quad$ is $\quad$ a finite-dimensional subspace of the complete space $L^{2}[0,1]$, and it is a complete basis for the Hilbert space $H$.

Proof. See[31].

Definition 2.5 Let $X=(X,\|\|$.$) be a normed space$ and let $Y$ be a subspace of $X$. Given a point $x \in X$, a point $\bar{y} \in Y$ is called a best approximation to $x$ out of $Y$ if $\bar{y}$ has the minimum distance from $x$. The problem of determining such a point is called the best approximation problem.

Ramark 2.6 If $f(t) \in L^{2}[0,1] \quad$ and $Y=\operatorname{Span}\left\{B_{0}^{n}(t), B_{1}^{n}(t), \cdots, B_{n}^{n}(t)\right\} \quad, \quad$ then the best approximation of order $n$ of the function $f(t)$ in $[0,1]$ is unique and is given by $P_{n}(t)$ where

$$
f(t) \simeq P_{n}(t)=C \varphi_{n}(t)
$$

and $C$ is completely dependent on $f(t)$. In fact $C$ can be obtained by 


$$
C=\left\langle f(t), \varphi_{n}(t)\right\rangle Q^{-1}
$$

where

$$
\begin{aligned}
& \left\langle f(t), \varphi_{n}(t)\right\rangle=\int_{0}^{1} f(t) \varphi_{n}^{T}(t) d t \\
& =\left[\begin{array}{llll}
\left\langle f(t), B_{0}^{n}(t)\right\rangle & \left\langle f(t), B_{1}^{n}(t)\right\rangle & \cdots & \left\langle f(t), B_{n}^{n}(t)\right\rangle
\end{array}\right],
\end{aligned}
$$

and the entries of the $(n+1) \times(n+1)$ matrix $Q$ are defined as follows:

$$
\begin{aligned}
Q & =\left\langle\varphi_{n}(t), \varphi_{n}(t)\right\rangle=\int_{0}^{1} \varphi_{n}(t) \varphi_{n}^{T}(t) d t \\
& =\int_{0}^{1}\left(\psi_{n} T_{n}(t)\right)\left(\psi_{n} T_{n}(t)\right)^{T} d t \\
& =\psi_{n} \int_{0}^{1} T_{n}(t) T_{n}^{T}(t) d t \psi_{n}^{T}=\psi_{n} G_{n} \psi_{n}^{T}
\end{aligned}
$$

where $G_{n}$ is the following $(n+1) \times(n+1)$ Hilbert matrix [31,32]:

$$
G_{n}=\left[\begin{array}{ccccc}
1 & \frac{1}{2} & \frac{1}{3} & \cdots & \frac{1}{n+1} \\
\frac{1}{2} & \frac{1}{3} & \frac{1}{4} & \cdots & \frac{1}{n+2} \\
\vdots & \vdots & \vdots & \ddots & \vdots \\
\frac{1}{n+1} & \frac{1}{n+2} & \frac{1}{n+3} & \cdots & \frac{1}{2 n+1}
\end{array}\right] .
$$

Theorem 2.7 Let $f(t) \in C^{m}[0,1]$; that is $f$ and all its derivatives up to order $m$ are continuous and differentiable on $[0,1]$ and let $Y=\operatorname{Span}\left\{B_{0}^{n}(t), B_{1}^{n}(t), \ldots, B_{n}^{n}(t)\right\}$. If $P_{n}(t)$ is the best approximation of $f$ out of $Y$, then the mean error bound is

$$
\left\|f(t)-P_{n}(t)\right\|_{2} \leq \frac{\sqrt{2} M}{(m) ! \sqrt{2 m+1}}
$$

where $M=\max _{t \in[0,1]}\left|f^{(m)}(t)\right|$.

Proof. We consider the Taylor polynomials

$$
\begin{aligned}
& \bar{f}(t)=f\left(t_{0}\right)+f^{\prime}\left(t_{0}\right)\left(t-t_{0}\right)+f^{\prime \prime}\left(t_{0}\right) \frac{\left(t-t_{0}\right)^{2}}{2} \\
& +\ldots+f^{(m-1)}\left(t_{0}\right) \frac{\left(t-t_{0}\right)^{m-1}}{(m-1) !}, \quad t, t_{0} \in[0,1]
\end{aligned}
$$

which we know that there exists $\epsilon \in[0,1]$ such that

$$
|f(t)-\bar{f}(t)|=\left|f^{(m)}(\epsilon)\right| \frac{\left(t-t_{0}\right)^{m}}{m !} .
$$

Since $P_{n}(t)$ is the best approximation of $f$, so we have

$$
\begin{aligned}
& \left\|f(t)-P_{n}(t)\right\|_{2}^{2} \leq\|f(t)-\bar{f}(t)\|_{2}^{2} \\
& =\int_{0}^{1}|f(t)-\bar{f}(t)|^{2} d t=\int_{0}^{1}\left(\left|f^{(m)}(\epsilon)\right| \frac{\left(t-t_{0}\right)^{m}}{m !}\right)^{2} d t \\
& \leq \frac{2 M^{2} S^{(2 m+1)}}{[(m) !]^{2}(2 m+1)} \leq \frac{2 M^{2}}{[(m) !]^{2}(2 m+1)}
\end{aligned}
$$

where $S=\max \left\{1-t_{0}, t_{0}\right\}$

Theorem 2.8 Suppose that $f(t) \in C^{m}[0,1]$; then for each $k \in \mathbb{N}, k \leq m$, and $k \leq n$, there exists an $(n+1) \times(n+1)$ matrix $D_{B_{n}}$ such that

$$
f^{(k)}(t) \simeq P_{n}^{(k)}(t)=C\left(D_{B_{n}}\right)^{k} \varphi_{n}(t)
$$

where $D_{B_{n}}=\psi_{n} \Lambda \mathcal{V}$, with

$$
\Lambda_{i+1, j+1}=\left\{\begin{array}{lc}
i, & i=j+1, \\
0 & \text { otherwise, }
\end{array}\right.
$$

for $i=0, \ldots, n$ and $j=0, \ldots, n-1$ and $\mathcal{V}$ can be expressed by

$$
\mathcal{V}_{k}=\psi_{n, k}^{-1}, \quad k=1,2, \ldots, n
$$

where $\psi_{n, k}^{-1}$ is $k^{\text {th }}$ row of $\psi_{n}^{-1}$.

Proof. See [33].

Theorem 2.9 Let $P_{B_{n}}$ be an $(n+1) \times(n+1)$ matrix; then

$$
\int_{0}^{x} \varphi_{n}(t) d t=P_{B_{n}} \varphi(x)
$$

where

$$
P_{B_{n}}=\mathcal{W} \Pi \mathcal{S}
$$

and $\mathcal{W}$ is defined as:

$$
\mathcal{W}=\left[\begin{array}{llll}
\mathcal{W}_{1} & \mathcal{W}_{2} & \cdots & \mathcal{W}_{n+1}
\end{array}\right]^{T}
$$

such that

$$
\begin{aligned}
& \mathcal{W}_{i}=\left[\begin{array}{lllll}
0 & 0 & \ldots & 0 & (-1)^{0}\left(\begin{array}{c}
n \\
i
\end{array}\right) \\
(-1)^{1}\left(\begin{array}{c}
n \\
i
\end{array}\right)\left(\begin{array}{c}
n-i \\
1
\end{array}\right) & \ldots & (-1)^{n-i}\left(\begin{array}{c}
n \\
i
\end{array}\right)\left(\begin{array}{c}
n-i \\
n-i
\end{array}\right)
\end{array}\right] .
\end{aligned}
$$


The $(n+1) \times(n+1)$ matrix $\Pi$ is defined as

$$
\Pi=\left[\begin{array}{ccccc}
1 & 0 & 0 & \cdots & 0 \\
0 & \frac{1}{2} & 0 & \cdots & 0 \\
\vdots & \vdots & \vdots & \ddots & \vdots \\
0 & 0 & 0 & \cdots & \frac{1}{n+1}
\end{array}\right]_{(n+1) \times(n+1)}
$$

and the matrix $\mathcal{S}$ is defined by

$$
\mathcal{S}=\left[\begin{array}{lllll}
\mathcal{W}_{2}^{-1} & \mathcal{W}_{3}^{-1} & \ldots & \mathcal{W}_{n+1}^{-1} & \mathcal{R}
\end{array}\right]^{T}
$$

where $\mathcal{R}$ is

$$
\mathcal{R}=\frac{Q^{-1}}{2 n+2}\left[\frac{\left(\begin{array}{l}
n \\
0
\end{array}\right)}{\left(\begin{array}{c}
2 n+1 \\
n+1
\end{array}\right)} \frac{\left(\begin{array}{l}
n \\
1
\end{array}\right)}{\left(\begin{array}{c}
2 n+1 \\
n+2
\end{array}\right)} \quad \cdots \frac{\left(\begin{array}{l}
n \\
n
\end{array}\right)}{\left(\begin{array}{c}
2 n+1 \\
2 n+1
\end{array}\right)}\right]^{T}
$$

and the elements of $Q$ are defined as

$$
Q_{(i+1)(j+1)}=\frac{\left(\begin{array}{c}
n \\
i
\end{array}\right)\left(\begin{array}{c}
n \\
j
\end{array}\right)}{(2 n+1)\left(\begin{array}{c}
2 n \\
i+j
\end{array}\right)}, \quad i, j=0,1, \cdots, n .
$$

The matrix $P_{B_{n}}$ is called the operational matrix of integration.

Proof. See [34].

Now assume that $w(x, t)$ is a function in a two-dimensional defined space on $[0,1] \times[0,1]$, and suppose that $\varphi_{n}(x)$ and $\varphi_{n}(t)$ are Bernstien vectors of degree $n$ over the interval [0,1]; then we can approximate $w(x, t)$ as follows:

$$
w(x, t) \simeq \sum_{i=0}^{n} \sum_{j=0}^{n} m_{i j} B_{i}^{n}(x) B_{j}^{n}(t)=\varphi_{n}^{T}(x) M_{w} \varphi_{n}(t)
$$

where

$$
M_{w}=\left[\begin{array}{ccccc}
m_{11} & m_{12} & \ldots & m_{1 n} & m_{1 n+1} \\
m_{21} & \ldots & \ldots & \ldots & m_{2 n+1} \\
\ldots & \ldots & \ddots & \ldots & \ldots \\
m_{n 1} & \ldots & \ldots & \ldots & m_{n n+1} \\
m_{n+11} & m_{n+12} & \ldots & m_{n+1 n} & m_{n+1 n+1}
\end{array}\right]
$$

and $m_{i j}, i, j=0,1,2, \cdots, n$, are constant numbers which are called control points. We describe later how one can find these control points.

\section{Problem Statement}

Now, due to (1), we consider the one-dimensional diffusion equation:

$$
w_{t}(x, t)-\lambda w_{x x}(x, t)=h(x, t), \quad(x, t) \in(0,1) \times(0,1)
$$

with the initial and boundary conditions as follows:

$$
\begin{aligned}
& w(x, 0)=f(x), \quad x \in[0,1] \\
& w(0, t)=g(t), \quad t \in[0,1]
\end{aligned}
$$

and the nonlocal boundary condition

$$
\int_{0}^{1} w(x, t) d x=u(t)
$$

where $h(x, t), f(x)$ and $g(t), \quad$ for $x \in[0,1]$ and $t \in[0,1]$, are given continuous and well-behaved functions. The parameter $\lambda$ is a given constant number.

We are going to find the control function $u(t)$ such that

$$
w(x, 1)=F(x)
$$

where $F(x)$ is a given continuous function for $x \in[0,1]$.

The control function $u(t)$ will be termed admissible if it is a measurable function on $[0,1]$ and the solution of the partial differential equation (9) satisfies the initial and boundary conditions (10)-(12) and also the terminal condition (13) holds.

The classical optimal control problem consists of finding an admissible control $u(t)$ which minimizes the functional

$$
J(u)=\int_{0}^{1}\|u(t)\|^{2} d t
$$

Non-local diffusion equation (9)-(11) arises in many scientific and engineering applications such as atmospheric pollution controls [35]. Efficient methods to solve diffusion equations are important in successful applications of these kind of problems. There are some methods in solving such problems (see[36] and reference therein). The present paper extends for the first time, the using of Bernstein's approximation in solving non-local diffusion optimal control problems.

To solve the optimal control problem (9)-(14), by using Bezier functions, one may convert the optimal control problem to a mathematical programming problem subject to algebraic equations.

According to the previous section, we assume that 


$$
w(x, t) \simeq \varphi_{n}^{T}(x) M_{w} \varphi_{n}(t)
$$

and that

$$
u(t) \simeq \sum_{i=0}^{n} u_{i} B_{i}^{n}(t)=M_{u} \varphi_{n}(t)
$$

where

$$
M_{u}=\left[\begin{array}{lllll}
u_{1} & u_{2} & u_{3} & \cdots & u_{n+1}
\end{array}\right]
$$

where $m_{i j}$ 's and $u_{k}$ 's, $i, j, k=1, \ldots, n+1$, are control points that need to be found. By applying (15) and (16), one can easily find that

$$
\begin{gathered}
w(x, 0) \simeq \varphi_{n}^{T}(x) M_{w} \varphi_{n}(0), \\
w(0, t) \simeq \varphi_{n}^{T}(0) M_{w} \varphi_{n}(t), \\
w(x, 1) \simeq \varphi_{n}^{T}(x) M_{w} \varphi_{n}(1), \\
w_{t}(x, t) \simeq \varphi_{n}^{T}(x) M_{w} D_{B_{n}} \varphi_{n}(t), \\
w_{x}(x, t) \simeq \varphi_{n}^{T}(x) D_{B_{n}}^{T} M_{w} \varphi_{n}(t), \\
w_{x x}(x, t) \simeq \varphi_{n}^{T}(x) D_{B_{n}}^{T} D_{B_{n}}^{T} M_{w} \varphi_{n}(t), \\
\int_{0}^{1} w(x, t) d x \simeq P_{B_{n}} \varphi_{n}^{T}(1) M_{w} \varphi_{n}(t) .
\end{gathered}
$$

We recall that the matrix $D_{B_{n}}$ is defined in (6), and for $\int_{0}^{1} w(x, t) d x$, we use (7).

Similarly for $J$, we have

$$
\begin{aligned}
& J(u)=\int_{0}^{1}\|u(t)\|^{2} d t \simeq \int_{0}^{1}\left[M_{u} \varphi_{n}(t) \varphi_{n}^{T}(t) M_{u}^{T}\right] d t \\
& =M_{u} \psi_{n} G_{n} \psi_{n}^{T} M_{u}^{T}
\end{aligned}
$$

where $G_{n}$ is given in (5).

Now the optimal control problem (9)-(13) with cost functional (14) can be approximated by the following optimization problem:

$$
\text { minimize } \quad J=M_{u} \psi_{n} G_{n} \psi_{n}^{T} M_{u}^{T}
$$

such that

$$
\begin{aligned}
& \varphi_{n}^{T}(x) M_{w} D_{B_{n}} \varphi_{n}(t)-\varphi_{n}^{T}(x) D_{B_{n}}^{2^{T}} M_{w} \varphi_{n}(t)=h(x, t), \\
& \varphi_{n}^{T}(x) M_{w} \varphi_{n}(0)=f(x), \\
& \varphi_{n}^{T}(0) M_{w} \varphi_{n}(t)=g(t), \\
& \varphi_{n}^{T}(1) P_{B_{n}}^{T} M_{w} \varphi_{n}(t)-M_{u} \varphi_{n}(t)=0, \\
& \varphi_{n}^{T}(x) M_{w} \varphi_{n}(1)=F(x)
\end{aligned}
$$

where $x, t \in[0,1]$ and the matrix $M_{w}$ and vector $M_{u}$ of order $n+1$ are unknowns. By solving the problem (19)-(20) one can find the matrix $M_{w}$ and the vector $M_{u}$. Thus by (15) and (16), it is possible to find the best approximation of functions $w(x, t)$ and $u(t)$, respectively. In the next section, we precisely describe how one can solve the optimization problem (19)-(20).

\section{Solving Optimization Problem}

Let $r, s \in \mathbb{Z}^{+}$. Choose $x_{i}$ 's $(i=0, \ldots, r)$ and $t_{j}$ 's $(j=0, \ldots, s)$ nodes on the interval $[0,1]$. Using these nodes in conditions (20), that convert these constraints into a algebraic system with $(s+1) \times(r+1)$ linear equations and $(n+1) \times(n+1)+(n+1)$ unknowns as:

$$
A_{i j} \mathcal{M}=b_{i j}
$$

where by (8) and (17),

$$
\mathcal{M}=\left[\begin{array}{llllll}
M_{w 0}^{T} & M_{w 1}^{T} & \ldots & M_{w(n-1)}^{T} & M_{w n}^{T} & M_{u}
\end{array}\right]^{T},
$$

and

$$
b_{i j}=\left[\begin{array}{lllll}
h\left(x_{i}, t_{j}\right) & f\left(x_{i}\right) & g\left(t_{j}\right) & 0 & F\left(x_{i}\right)
\end{array}\right]^{T},
$$

and $A_{i j}$ is a $5 \times(n+1)(n+2)$ matrix defined by discrete equations (20) for every $i \in\{0, \ldots, r\}$ and $j \in\{0, \ldots, s\}$.

Now we solve a quadratic programming problem to find $\mathcal{M}$ (in other words, $M_{w}$ and $M_{u}$ ):

The algorithm of this method is as follows:

INPUT: $n, r$ and $s$.

Calculate $D_{n}$ and $P_{B_{n}}$, operational matrices of derivative and integral.

Set $M_{w}$ and $M_{u}$, control points matrix and vector for $w(x, t)$ and $u(t)$, respectively.

For $i=0$ to $r$ and $j=0$ to $s$ do: Compute $A_{i j}$ and $b_{i j}$, for the linear constraints of problem.

Solve the following quadratic programming problem to find $M_{w}$ and $M_{u}$.

$$
\text { minimize } \quad J=M_{u} \psi_{n} G_{n} \psi_{n}^{T} M_{u}^{T}
$$

Such that $A_{i j} \mathcal{M}=b_{i j}, \quad i=0,1, \ldots, r, \quad j=0,1, \ldots, s$.

OUTPUT: $w(x, t) \simeq \varphi_{n}^{T}(x) M_{w} \varphi_{n}(t), u(t) \simeq M_{u} \varphi_{n}(t)$, and cost function $J$. 
By using different optimization tools, this optimization problem can be solved by many useful computational softwares such as MATLAB. We need to remind

- $x_{0}=t_{0}=0$ and $x_{r}=t_{s}=1$.

- Generally $x_{i}$ 's $(i=1, \ldots, r-1)$ and $t_{j}$ 's $(i=1, \ldots, s-1)$ are not equal in lengths.

- If the solution is undesired, then we may increase $r$ or $s$ (the number of nodes) or $n$ (the degree of Bezier function).

- The $(n+1) \times(n+1)$ matrix $\psi_{n} G_{n} \psi_{n}^{T}$ is symmetric positive definite.

\section{Convergence Analysis}

In this section, we provide a theoretical analysis about the convergence of above method. Without loss of generality, for the functional $J$ in (14), consider $\|u(t)\|^{2}=u(t) u^{T}(t)$. Also for simplicity, consider $\lambda=1$. Summing two relations (9) and (12), we have

$$
w_{t}(x, t)-w_{x x}(x, t)+\int_{0}^{1} w(x, t) d x-u(t)=h(x, t) .
$$

We prove the convergence by the degree raising of the Bezier functions approximation when the control-point-method is applied to the following optimal control problem:

$$
\text { minimize } \quad J=\int_{0}^{1} u(t) u^{T}(t) d t
$$

subject to

$$
\begin{aligned}
& L\left(w(x, t), w_{t}(x, t), w_{x x}(x, t), u(t)\right)=w_{t}(x, t) \\
& -w_{x x}(x, t)+\int_{0}^{1} w(x, t) d x-u(t)=h(x, t)
\end{aligned}
$$

where $\quad w(x, 0)=f(x), \quad w(0, t)=g(t) \quad, \quad$ and $w(x, 1)=F(x)$.

Definition 5.1 Let $\Omega=[0,1] \times[0,1]$ and let $\mathcal{P}=\left\{B_{i}^{n}(x) B_{j}^{m}(t) \mid 0 \leq i \leq n, 0 \leq j \leq m\right\} \subset L^{2}(\Omega)$ be the set of Bernstein polynomial products when $n, m \in \mathrm{N} \cup\{0\}$. We define $\mathfrak{B}_{n m}$ as $\mathfrak{B}_{n m}=\operatorname{Span}\{\mathcal{P}\}$.

Theorem 5.2 Let $P_{n m}$ be the best approximation of $f \in L^{2}(\Omega)$ in $\mathfrak{B}_{n m}$. If

$$
s_{n m}(f)=\int_{\Omega}\left|f(x, t)-P_{n m}(x, t)\right|^{2} d \Omega,
$$

then

$$
\lim _{(n, m) \rightarrow(\infty, \infty)} s_{n m}(f)=0 .
$$

Proof. See [37].

Theorem 5.3 Under the stated assumptions Theorem 5.2, the approximate solutions $B_{n m}=\varphi_{m}^{T}(x) M_{w} \varphi_{n}(t)$ and $u_{n}=M_{u} \varphi_{n}(t)$, convergence, respectively, to the exact solutions $w^{*}(x, t)$ and $u^{*}(t)$ in the optimal control problem (22)-(23) when $(n, m) \rightarrow(\infty, \infty)$.

Proof. Let $\epsilon>0$ and let $(x, t) \in[0,1] \times[0,1]$. By using Theorem 5.2, we can easily find the Bernstein functions $P_{n_{1} m_{1}}$ and $w_{n_{1}}$ such that and $\left\|w^{*}(x, t)-P_{n_{1} m_{1}}(x, t)\right\| \leq \frac{\epsilon}{24}$,

$\left\|u^{*}(t)-w_{n_{1}}(t)\right\| \leq \frac{\epsilon}{24}$.

By assuming $n_{1}$ and $m_{1}$ sufficiently large, it is easy to find that

$$
\begin{aligned}
& \left\|f(x)-P_{n_{1} m_{1}}(x, 0)\right\| \leq \frac{\epsilon}{24}, \\
& \left\|g(t)-P_{n_{1} m_{1}}(0, t)\right\| \leq \frac{\epsilon}{24}, \\
& \left\|F(x)-P_{n_{1} m_{1}}(x, 1)\right\| \leq \frac{\epsilon}{24} .
\end{aligned}
$$

But perhaps $P_{n_{1} m_{1}}(x, t)$ and $w_{n_{1}}(t)$ do not satisfy precisely in the initial and boundary conditions. So we define $B_{n_{1} m_{1}}(x, t)$ as follows:

$$
B_{n_{1} m_{1}}(x, t)=P_{n_{1} m_{1}}(x, t)+x t \alpha(x)+x \beta(x)+\gamma(t)
$$

such that this new function satisfies the initial and boundary conditions, that is,

$$
\begin{aligned}
& B_{n_{1} m_{1}}(x, 0)=f(x), \\
& B_{n_{1} m_{1}}(0, t)=g(t), \\
& B_{n_{1} m_{1}}(x, 1)=F(x) .
\end{aligned}
$$

According to (23), we have

$$
\begin{aligned}
& \left\|f(x)-P_{n_{1} m_{1}}(x, 0)\right\|=\|x \beta(x)+\gamma(0)\| \leq \frac{\epsilon}{24}, \\
& \left\|g(t)-P_{n_{1} m_{1}}(0, t)\right\|=\|\gamma(t)\| \leq \frac{\epsilon}{24}, \\
& \left\|F(x)-P_{n_{1} m_{1}}(x, 1)\right\|=\|x \alpha(x)+x \beta(x)+\gamma(1)\| \leq \frac{\epsilon}{24} .
\end{aligned}
$$

Since

$\|x \beta(x)\|-\|\gamma(0)\| \leq\|x \beta(x)+\gamma(0)\| \leq \frac{\epsilon}{24}$,

$\|x \alpha(x)\|-\|x \beta(x)+\gamma(1)\| \leq\|x \alpha(x)+x \beta(x)+\gamma(1)\| \leq \frac{\epsilon}{24}$, 
and $\|\gamma(t)\| \leq \frac{\epsilon}{24}$ for all $t \in[0,1]$, we have

$$
\|x \beta(x)\| \leq \frac{\epsilon}{24}+\|\gamma(0)\| \leq \frac{\epsilon}{24}+\frac{\epsilon}{24}=\frac{\epsilon}{12},
$$

also

$$
\begin{aligned}
& \|x \alpha(x)\| \leq \frac{\epsilon}{24}+\|x \beta(x)+\gamma(1)\| \\
& \leq \frac{\epsilon}{24}+\|x \beta(x)\|+\|\gamma(1)\| \leq \frac{\epsilon}{24}+\frac{\epsilon}{24}+\frac{\epsilon}{24}=\frac{\epsilon}{8} .
\end{aligned}
$$

Hence

$$
\begin{aligned}
& \left\|B_{n_{1} m_{1}}(x, t)-w^{*}(x, t)\right\| \\
& =\left\|P_{n_{1} m_{1}}(x, t)+x t \alpha(x)+x \beta(x)+\gamma(t)-w^{*}(x, t)\right\| \\
& \leq\left\|P_{n_{1} m_{1}}(x, t)-w^{*}(x, t)\right\|+\|t\|\|x \alpha(x)\|+\|x \beta(x)\|+\|\gamma(t)\| \\
& \leq \frac{\epsilon}{24}+\frac{\epsilon}{8}+\frac{\epsilon}{12}+\frac{\epsilon}{24}=\frac{7 \epsilon}{24}<\frac{\epsilon}{3} .
\end{aligned}
$$

Consequently,

$$
B_{n_{1} m_{1}}(x, t)=\varphi_{n_{1}}^{T}(x) M_{1} \varphi_{m_{1}}(t) \simeq w^{*}(x, t)
$$

and

$$
\begin{aligned}
& \frac{\partial w^{*}(x, t)}{\partial t} \simeq \frac{\partial P_{n_{1} m_{1}}(x, t)}{\partial t}+x \alpha(x)+\gamma^{\prime}(t) \simeq \varphi_{n_{2}}^{T}(x) M_{2} \varphi_{m_{2}}(t), \\
& \frac{\partial^{2} w^{*}(x, t)}{\partial x^{2}} \simeq \frac{\partial^{2} P_{n_{1} m_{1}}(x, t)}{\partial x^{2}}+x t \alpha^{\prime \prime}(x)+2 t \alpha^{\prime}(x) \\
& +2 \beta^{\prime}(x)+x \beta^{\prime \prime}(x) \simeq \varphi_{n_{3}}^{T}(x) M_{3} \varphi_{m_{3}}(t), \\
& \int_{0}^{1} w^{*}(x, t) d x \simeq M_{4} \varphi_{m_{4}}(t), \\
& u^{*}(t) \simeq M_{5} \varphi_{m_{5}}(t) .
\end{aligned}
$$

By Theorem 5.2, one can increase $n_{1}, m_{1}, n_{2}, m_{2}, n_{3}, m_{3}, m_{4}$, and $m_{5}$ such that

$$
\begin{aligned}
& \left\|\frac{\partial w^{*}(x, t)}{\partial t}-\varphi_{n_{2}}^{T}(x) M_{2} \varphi_{m_{2}}(t)\right\| \leq \frac{\epsilon}{24}, \\
& \left\|\frac{\partial^{2} w^{*}(x, t)}{\partial x^{2}}-\varphi_{n_{3}}^{T}(x) M_{3} \varphi_{m_{3}}(t)\right\| \leq \frac{\epsilon}{24}, \\
& \left\|\int_{0}^{1} w^{*}(x, t) d x-M_{4} \varphi_{m_{4}}(t)\right\| \leq \frac{\epsilon}{24}, \\
& \left\|u^{*}(t)-M_{5} \varphi_{m_{5}}(t)\right\| \leq \frac{\epsilon}{24} .
\end{aligned}
$$

Thus for every $n \geq N=\max \left\{n_{1}, n_{2}, n_{3}\right\} \quad$ and $m \geq M=\max \left\{m_{1}, m_{2}, m_{3}, m_{4}, m_{5}\right\}$, we define

$$
\begin{aligned}
& L\left(B_{n m}(x, t), B_{n m_{t}}(x, t), B_{n m_{x x}}(x, t), \eta_{n}(t)\right) \\
& =\varphi_{n}^{T}(x) M_{2} \varphi_{m}(t)-\varphi_{n}^{T}(x) M_{3} \varphi_{m}(t)+M_{4} \varphi_{m}(t)-M_{5} \varphi_{m}(t) .
\end{aligned}
$$

Also (22) implies

$$
\begin{aligned}
& \left\|h(x, t)-L\left(B_{n m}(x, t), B_{n m_{t}}(x, t), B_{n m_{x x}}(x, t), \eta_{n}(t)\right)\right\| \\
& =\| L\left(w^{*}(x, t), w_{t}^{*}(x, t), w_{x x}^{*}(x, t), u^{*}(t)\right) \\
& -L\left(B_{n m}(x, t), B_{n m_{t}}(x, t), B_{n m_{x x}}(x, t), \eta_{n}(t)\right) \| \\
& \leq\left\|w_{t}^{*}(x, t)-\varphi_{n}^{T}(x) M_{2} \varphi_{m}(t)\right\|+\left\|w_{x x}^{*}(x, t)-\varphi_{n}^{T}(x) M_{3} \varphi_{m}(t)\right\| \\
& +\left\|\int_{0}^{1} w^{*}(x, t) d x-M_{4} \varphi_{m}(t)\right\|+\left\|u^{*}(t)-M_{5} \varphi_{m}(t)\right\| \\
& \leq\left\|w_{t}^{*}(x, t)-\varphi_{n}^{T}(x) M_{2} \varphi_{m}(t)\right\| \\
& +\left\|\int_{0}^{1} w^{*}(x, t) d x-M_{4} \varphi_{m}(t)\right\|+\left\|u^{*}(t)-M_{5} \varphi_{m}(t)\right\| \\
& \leq \frac{\epsilon}{24}+\frac{\epsilon}{24}+\frac{\epsilon}{24}+\frac{\epsilon}{24}=\frac{\epsilon}{6} .
\end{aligned}
$$

Thus $B_{n m}(x, t)$ and $\eta_{n}(t)$ that approximately satisfy the diffusion equation (9) with initial and boundary conditions (10)-(13) tend to $w^{*}(x, t)$ and $u^{*}(t)$ respectively, as $n$ and $m$ tend to infinity.

Theorem 5.4 Suppose that $\mathbb{U}$ is a set of all admissible controls $u(t)$ for the optimal control problem (21)-(22), that $\mu_{n}$ is the minimum of the functional $J$ on (19), and that $\mu$ is the minimum of the functional $J$ on $\mathbb{U}$. Then $\mu_{n} \rightarrow \mu$ as $n$ tends to infinity.

Proof. Let $\epsilon>0$ and let $u^{*} \in \mathbb{U}$ such that $J\left(u^{*}\right)<\mu+\epsilon$. Since $J$ is continuous, if $\left|u-u^{*}\right|<\delta$, then $\left|J(u)-J\left(u^{*}\right)\right|<\epsilon$. By using Theorem 2.7 for a sufficiently large value of $n$, there exists $\eta_{n}(t)$ such that $\left|\eta_{n}-u^{*}\right|<\delta$, then

$$
\begin{aligned}
& \mu \leq \mu_{n}=\left|J\left(\eta_{n}\right)-J\left(u^{*}\right)+J\left(u^{*}\right)\right| \\
& \leq\left|J\left(\eta_{n}\right)-J\left(u^{*}\right)\right|+\left|J\left(u^{*}\right)\right| \leq \mu+2 \epsilon
\end{aligned}
$$

where $\mu_{n}=J\left(u_{n}\right)$. Because $\epsilon$ is arbitrary, this completes the proof.

\section{NUMERICAL EXAMPLES}

In this section, to validate the accuracy of the presented method, three examples are considered. These test examples are solved by using powerful MATLAB 2017a software on an Intel Core i5-4200U.

\section{Example 1}

Consider the following optimal control problem (see [38]) 


$$
\text { minimize } \quad J(u)=\int_{0}^{1}\|u(t)\|^{2} d t
$$

such that

$$
\begin{aligned}
& w_{t}(x, t)-w_{x x}(x, t)=0, \quad(x, t) \in(0,1) \times(0,1), \\
& w_{x}(0, t)=u(t), \\
& w_{x}(1, t)=0, \quad w(x, 0)=\cos (\pi x)
\end{aligned}
$$

where the function $\|\cdot\|$ indicates the Euclidean norm. We are going to find the admissible control function $u($.$) such that the solution of the above partial$ differential equation corresponding to the given boundary conditions satisfies the following terminal condition:

$$
w(x, 1)=\cos (\pi x) \exp \left(-\pi^{2}\right)
$$

The exact solution is $u(t)=0$ and $w(x, t)=\cos (\pi x) \exp \left(-\pi^{2} t\right)$. By using the Bezier function, we expect

$$
u(t)=M_{u} \varphi_{n}(t) \simeq 0
$$

and

$$
w(x, t)=\varphi_{n}^{T}(x) M_{w} \varphi_{n}(t) \simeq \cos (\pi x) \exp \left(-\pi^{2} t\right) .
$$

For $n=8$, the control is found as

$$
u(t) \simeq 6.0695 \times 10^{-9} B_{0}^{8}(t)-3.6448 \times 10^{-8} B_{1}^{8}(t)
$$$$
+1.1633 \times 10^{-7} B_{2}^{8}(t)-2.2266 \times 10^{-7} B_{3}^{8}(t)
$$$$
+2.7047 \times 10^{-7} B_{4}^{8}(t)-2.1110 \times 10^{-7} B_{5}^{8}(t)
$$$$
+1.0286 \times 10^{-7} B_{6}^{8}(t)-2.8404 \times 10^{-8} B_{7}^{8}(t)+3.2833 \times 10^{-9} B_{8}^{8}(t),
$$

and the terminal condition is found as

$$
\begin{aligned}
& w(x, 1) \simeq 5.1723 \times 10^{-5} B_{0}^{8}(x)+4.1379 \times 10^{-4} B_{1}^{8}(x) \\
& +1.1929 \times 10^{-3} B_{2}^{8}(x)+1.3657 \times 10^{-3} B_{3}^{8}(x) \\
& -9.8504 \times 10^{-9} B_{4}^{8}(x)-1.3657 \times 10^{-3} B_{5}^{8}(x) \\
& -1.1929 \times 10^{-3} B_{6}^{8}(x)-4.1379 \times 10^{-4} B_{7}^{8}(x)-5.1723 \times 10^{-5} B_{8}^{8}(x),
\end{aligned}
$$

and

$$
J=9.6549 \times 10^{-19} .
$$

The absolute error of the exact solution and approximate solution given for different values of the degree of Bezier polynomials are shown in Table 1 . The numerical results in this table show that the present method is convergence. To illustrate this aim, we define

$$
e_{n}=\max _{i \in\{0, \ldots, r\}}\left|w\left(x_{i}, 1\right)-\bar{w}\left(x_{i}, 1\right)\right|
$$

where $\bar{w}(x, 1)$ indicates the approximate solution of the diffusion equation at the final time $t=1$.

Table 1. The absolute error in Example 1.

\begin{tabular}{|c|c|}
\hline Degree of Bezier polynomial $(n)$ & Absolute error $\left(e_{n}\right)$ \\
\hline 10 & $2.1866 \times 10^{-12}$ \\
\hline 11 & $2.6110 \times 10^{-15}$ \\
\hline 13 & $2.1460 \times 10^{-17}$ \\
\hline 15 & $1.9590 \times 10^{-17}$ \\
\hline 16 & $1.3922 \times 10^{-17}$ \\
\hline
\end{tabular}

Fig.1 and Fig.2 show the approximated graphs of control and terminal condition $w(x, 1)$.

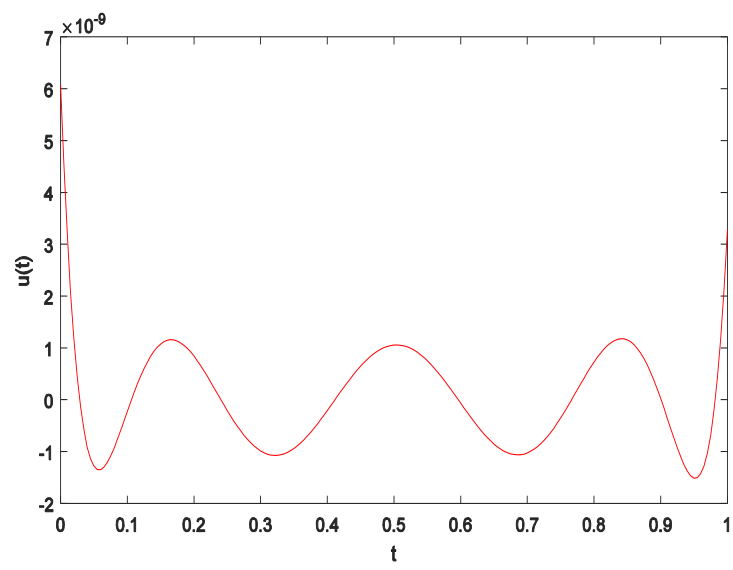

Fig.1. The graph of approximated control

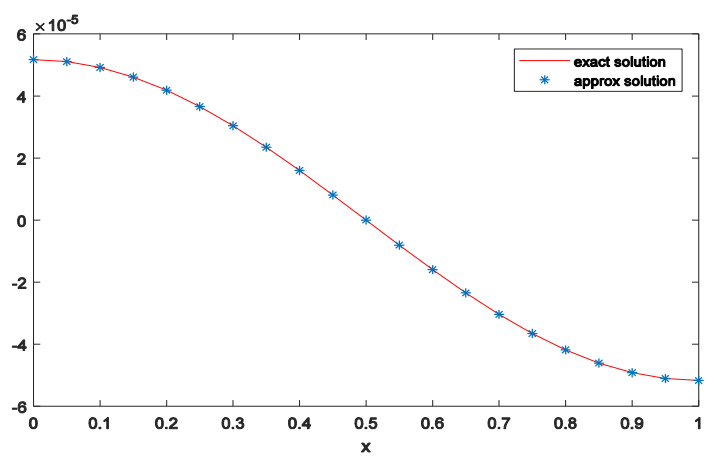

Fig.2. The graphs of approximated and exact terminal trajectory $w(x, 1)$

\section{Example 2}

In the second example, we consider the one-dimensional diffusion equation (9)-(13), where (see [39]):

$$
\begin{aligned}
& h(x, t)=0, \quad f(x)=\cos \left(\frac{\pi x}{2}\right), \\
& g(t)=\exp \left(-\frac{\pi^{2}}{4} t\right), \quad F(x)=\cos \left(\frac{\pi x}{2}\right) \exp \left(-\frac{\pi^{2}}{4}\right) .
\end{aligned}
$$


Now we need to find $u($.$) and minimize the$ functional

$$
J(u)=\int_{0}^{1}\|u(t)\|^{2} d t
$$

By using the Bernstein polynomial of degree 8 , the control function is found as

$$
\begin{aligned}
& u(t) \simeq 0.6366 B_{0}^{8}(t)-2.5370 B_{1}^{8}(t)+5.8914 B_{2}^{8}(t) \\
& -8.7804 B_{3}^{8}(t)+8.7012 B_{4}^{8}(t)-5.7216 B_{5}^{8}(t)+2.3956 B_{6}^{8}(t) \\
& -0.5706 B_{7}^{8}(t)+0.0540 B_{8}^{8}(t),
\end{aligned}
$$

and the trajectory $w(x, 1)$ is found as:

$$
\begin{aligned}
& w(x, 1) \simeq 8.4805 \times 10^{-2} B_{0}^{8}(x)+8.4805 \times 10^{-2} B_{1}^{8}(x) \\
& +8.1068 \times 10^{-2} B_{2}^{8}(x)+7.3595 \times 10^{-2} B_{3}^{8}(x) \\
& +6.2693 \times 10^{-2} B_{4}^{8}(x)+4.8976 \times 10^{-2} B_{5}^{8}(x) \\
& +3.3303 \times 10^{-2} B_{6}^{8}(x)+1.6651 \times 10^{-2} B_{7}^{8}(x) .
\end{aligned}
$$

The cost function $J$ is

$$
J=5.0070 \times 10^{-3}
$$

Table 2. The absolute error in Example 2.

\begin{tabular}{|c|c|}
\hline Degree of Bezier polynomial $(n)$ & Absolute error $\left(e_{n}\right)$ \\
\hline 8 & $6.9440 \times 10^{-10}$ \\
\hline 9 & $2.2657 \times 10^{-11}$ \\
\hline 10 & $8.1517 \times 10^{-13}$ \\
\hline 11 & $2.3004 \times 10^{-13}$ \\
\hline 12 & $5.7024 \times 10^{-14}$ \\
\hline
\end{tabular}

Fig. 3 and Fig.4 show the approximated graphs of control and terminal condition $w(x, 1)$.

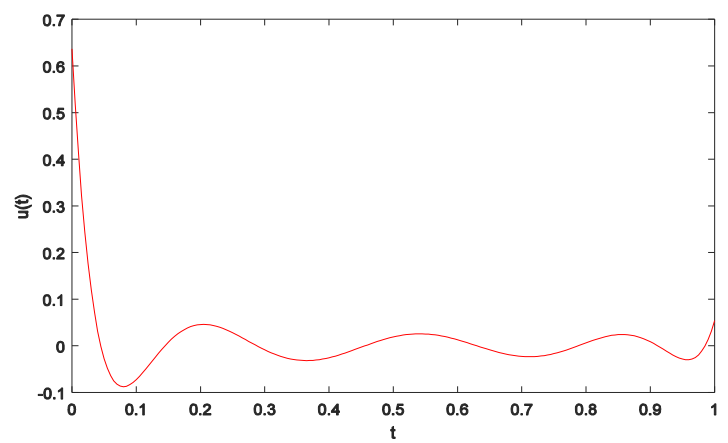

Fig.3. The graph of approximated control

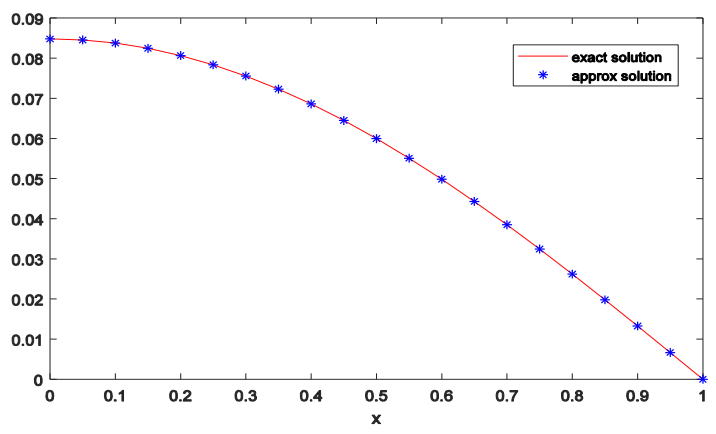

Fig.4. The graphs of approximated and exact terminal trajectory $w(x, 1)$

\section{Example 3}

In this example, we consider the one-dimensional diffusion equation (9)-(13) where (see [40]):

$$
\begin{aligned}
& h(x, t)=\left(x^{2}-2\right) e^{t}, \quad f(x)=x^{2}, \\
& g(t)=0, \quad F(x)=x^{2} e .
\end{aligned}
$$

Now we must find $u($.$) and minimize the functional$

$$
J(u)=\int_{0}^{1}\|u(t)\|^{2} d t .
$$

The exact solution is $u(t)=\frac{e^{t}}{3}$ and $w(x, t)=x^{2} e^{t}$.

For $n=8$ the control of this problem is found as

$$
\begin{aligned}
& u(t) \simeq 0.3333 B_{0}^{8}(t)-0.7468 B_{1}^{8}(t)-0.1095 B_{2}^{8}(t) \\
& -0.0346 B_{3}^{8}(t)+0.1195 B_{4}^{8}(t)+0.1189 B_{5}^{8}(t) \\
& -0.1559 B_{6}^{8}(t)-0.3836 B_{7}^{8}(t)+0.9061 B_{8}^{8}(t),
\end{aligned}
$$

and the terminal condition is approximated by

$$
\begin{aligned}
& w(x, 1) \simeq 2.7183 B_{2}^{8}(x)+16.3097 B_{3}^{8}(x) \\
& +40.7742 B_{4}^{8}(x)+54.3656 B_{5}^{8}(x)+40.7742 B_{6}^{8}(x) \\
& +16.3097 B_{7}^{8}(x)+2.7183 B_{8}^{8}(x),
\end{aligned}
$$

and $J=10.1825 \times 10^{-3}$.

Fig.5 and Fig. 6 show the approximated graphs of control and terminal condition $w(x, 1)$.

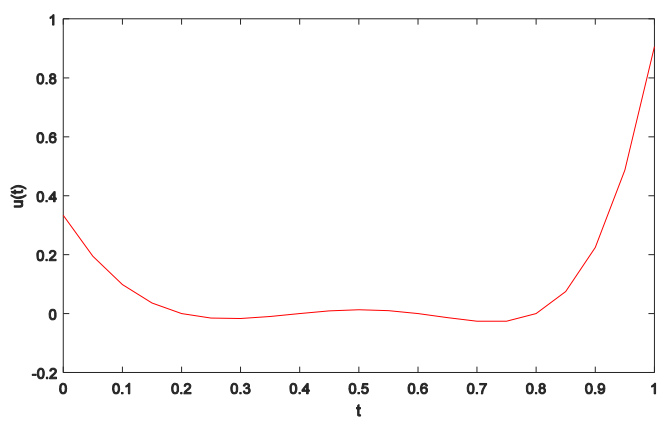

Fig.5. The graph of approximated control 


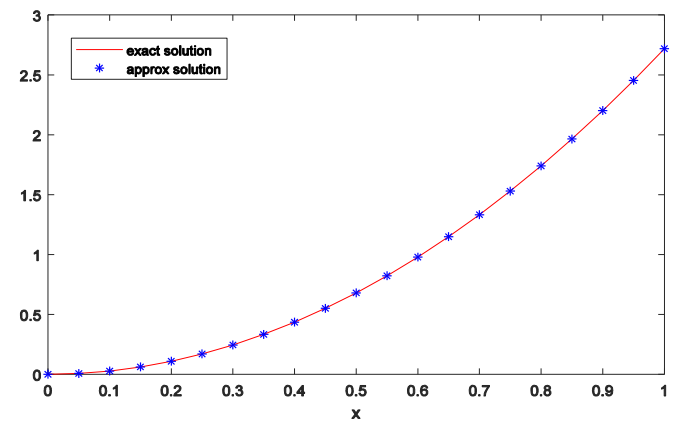

Fig.6. The graphs of approximated and exact terminal trajectory $w(x, 1)$

\section{CONCLUSION}

In this paper, we introduced a new set of functions that are called Bezier functions for solving optimal control problems governed by a nonlocal diffusion equation with given boundary and terminal conditions. The method is general and easy to implement by using operational matrices. Operational matrices, together with the collocation method, were used to approximate solution of this kind problems. The convergence of the method was proved. Some numerical examples were given to illustrate that this method is efficient.

\section{ACKNOWLEDGEMENT}

We would also like to show our gratitude to the referees for their helpful comments and useful suggestions.

\section{REFERENCES}

[1] P. M. Morse and H. Feshbach, "Methods of Theoretical Physics," New York, McGraw-Hill, 1953.

[2] J. C. Maxwell, "On the Dynamic Theory of Gases," Phil. Trans. R. Soc., vol. 157, pp. 49-88, 1967.

[3] H. S. Carslaw and J. C. Jaeger, "Conduction of Heat in Solids," Oxford University Press, pp. 96-97, 1959.

[4] Z. Rotem and J. E. Neilson, "Exact Solution for Diffusion to Flow Down an Incline," Can. J. Chem. Engng. Sci., vol. 47, pp. 341-346, 1969

[5] A. Tamir and Y. Taitel, "Diffusion to flow down an incline with surface resistance," Chemical Engineering Science, vol. 26, pp. 799-808, 1971.

[6] Y. Taitel, "On the parabolic, hyperbolic and discrete formulation of the heat conduction equation," Int. J. Heat Mass Transfer, vol. 15, pp. 369-371, 1972.

[7] V. P. Pavlov and V. M. Kudoyarova, "Spline based numerical method for Heat conduction nonlinear problems solution," Procedia Engineering, vol. 206, pp. 704-709, 2017.

[8] K. Al-Khaled, "Finite Fourier transform for solving potential and steady-state temperature problems," Advances in Difference Equations, vol. 1, pp. 1-14, 2018.

[9] R. W. Lewis, P. Nithiarasu and K. N. Seetharamu, "Fundamentals of the Finite Element Method for Heat and Fluid Flow," John Wiley and Sons. Ltd., 2005.

[10] E. Li, Z. Zhang, Z. C. He, X. Xu, G. R. Liu and et al. "Smoothed finite element method with exact solutions in heat transfer problems," International Journal of Heat and
Mass Transfer, vol. 78, pp. 1219-1231, 2014.

[11] L. Su and T. Jiang, "Numerical Method for Solving Nonhomogeneous Backward Heat Conduction Problem," International Journal of Differential Equations, vol. 2, pp. 1-11, 2018.

[12] C. R. Jun and G. H. Xia, "Meshless analysis of three-dimensional steady-state heat conduction problems," Chin. Phys. B, vol. 19, pp. 1-7, 2010.

[13] I. V. Singh, M. Tanaka and M. Endo, "Meshless method for nonlinear heat conduction analysis of nano-composites," Heat Mass Transfer, vol. 43, pp. 1097-1106, 2007.

[14] A. Chakrabarti and S. C. Martha, "Approximate solutions of Fredholm integral equations of the second kind," Appl. Math. Comput., vol. 211, pp. 459-466, 2009.

[15] F. Ghomanjani, M. H. Farahi and A. Kilicman, "Bezier curves for solving Fredholm integral equations of the second kind," Mathematical Problems in Engineering, pp. $1-6,2014$.

[16] B. N. Mandal and S. Bhattacharya, "Numerical solution of some classes of integral equations using Bernstein polynomials," Appl. Math. Comput., vol. 190, pp. 1707-1716, 2007.

[17] S. Bhattacharya and B. N. Mandal, "Use of Bernstein polynomials in numerical solutions of Volterra integral equations," Appl. Math. Sci., vol. 2, pp. 1773-1787, 2008

[18] K. Maleknejad, E. Hashemizadeh and B. Basirat, "Computational method based on Bernstein operational matrices for nonlinear Volterra-Fredholm-Hammerstein integral equations," Commun. Nonl. Sci. Number. Simul., vol. 17, pp. 52-61, 2012.

[19] J. Zheng, T. W. Sederberg and R. W. Johnson, "Least squares methods for solving differential equations using Bezier control points," Applied Numerical Mathematics, vol. 48, pp. 237-252, 2004.

[20] E. Safaie and M. H. Farahi, "An approximate method for solving fractional TBVP with state delay by Bernstein polynomials," Comp. Appl. Math., vol. 298, pp. 2-16, 2016.

[21] R. Winkel, "Generalized Bernstein polynomials and Bezier curves: an application of umbral calculus to computer aided geometric design," Advances in Applied Mathematics, vol. 27, pp. 51-81, 2001.

[22] F. Ghomanjani and M. H. Farahi, "Optimal Control of Switched Systems based on Bezier Control Points," I.J. Intelligent Systems and Applications, vol. 7, pp. 16-22, 2012.

[23] M. Ainsworth and G. Fu, "Bernstein-Bezier Bases for Tetrahedral Finite Elements," Comput. Methods Appl. Mech. Engrg., vol. 340, pp. 178-201, 2018.

[24] P. P. C. Alzate, C. A. R. Varela and F. Mesa, "Application of Bezier curves to obtain a band of certainty with noise contaminated data," Contemporary Engineering Sciences, vol. 11, pp. 4905-4912, 2018.

[25] F. Ghomanjani, M. H. Farahi, A. Kilicman, A. V. Kamyad and N. Pariz, "Bezier curves based numerical solutions of deley systems with inverse time," Mathematical Problems in Engineering, vol. 1, pp. 1-16, 2014.

[26] R. Janssen, S. R. Moisik and D. Dediu, "Modelling human hard palate shape with Bezier curves," PLoS ONE, vol. 13, pp. 1-38, 2018.

[27] Y. Jin, S. Zhao and Y. Wang, "An Optimal Feed Interpolator Based on G2 Continuous Bezier Curves for High-Speed Machining of Linear Tool Path," Jin et al. Chin. J. Mech. Eng., vol. 32, pp. 32-43, 2019.

[28] R. Palomar, J. Gomez-Luna, F. Cheikh, J. Olivares-Bueno and O. Elle, "High-Performance Computation 
of Bezier Surfaces on Parallel and Heterogeneous Platforms," International Journal of Parallel Programming, vol. 46, pp. 1035-1062, 2018.

[29] R. Szabo and A. Gontean, "Photovoltaic cell and module I-V characteristic approximation using bezier curves," Appl. Sci., vol 8, pp. 1-23, 2018.

[30] M. Alipour, D. Rostamy and D. Baleanu, "Solving multi-dimensional fractional optimal control problems with inequality constraint by Bernstein polynomials operational matrices," J. Vib. Control, vol. 19, pp. 2523-2540, 2012.

[31] E. Kreyszig, "Introduction to Functional Analysis with Applications," New York: Wiley, 1978.

[32] M. Dehghan, S. A. Yousefi and K. Rashedi, "Ritz-Galerkin method for solving an inverse heat conduction problem with a nonlinear source term via Bernstein multi-scaling functions and cubic B-spline functions," Inverse Problems in Science and Engineering, vol. 21, pp. 500-523, 2013.

[33] K. Karimi, M. Alipour and M. Khaksarfard, "Numerical solution of nonlocal parabolic partial differential equation via Bernstein polynomial method," Journal of Mathematics, vol. 48, pp. 47-53, 2016.

[34] S. A. Yousefi and M. Behroozifar, "Operational matrices of Bernstein polynomials and their applications," I. J. Sys. Sci., vol. 41, pp. 709-716, 2010.

[35] H. Fu, "A characteristic finite element method for optimal control problems governed by convection-diffusion equations," Journal of Computational and Applied Mathematics, vol. 235, pp. 825-836, 2010.

[36] A. Lapin and E. Laitinen, "Efficient Iterative Method for Solving Optimal Control Problem Governed by Diffusion Equation with Time Fractional Derivative," Lobachevskii Journal of Mathematics, vol. 40, pp. 479-488, 2019.

[37] T. J. Rivlin, "An Introduction to the Approximation of the Functions," New York: Dover, 1969.

[38] J. E. Rubio, "Control and optimization the linear treatment of nonlinear problems," Manchester University Press, 1986.

[39] M. Tatari and M. Dehghan, "On the solution of the nonlocal parabolic partial differential equations via radial basis functions," Appl. Math. Model, vol. 33, pp. 1729-1738, 2009.

[40] M. Bastani and N. K. Salkuyeh, "Numerical studies of a nonlocal parabolic partial differential equations by spectral collocation method with preconditioning," Computational Mathematics and Modeling. vol. 24, pp. 81-89, 2013.

\section{Authors' Profiles}

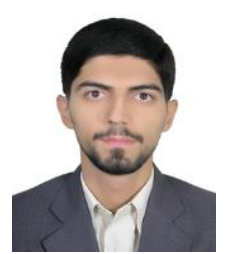

Ali Ketabdari is a Ph.D. student in control theory and optimization at the Department of Applied Mathematics, Ferdowsi University of Mashhad, Iran. He received his B.S and M.Sc respectively in Applied Mathematics and control theory from the Ferdowsi University of Mashhad in 2009, 2011. His scientific interests include optimal control, optimization, delay differential equations, and fractional calculus.

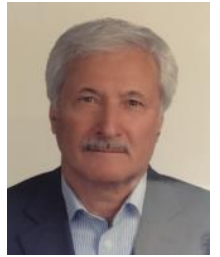

Mohammad Hadi Farahi is a Professor at the Department of Applied Mathematics, School of Mathematics, Ferdowsi University of Mashhad, Iran. He obtained his B.S, M.Sc and $\mathrm{Ph} . \mathrm{D}$. respectively from the Ferdowsi University of Mashhad, Iran in 1972, Brunel University, UK in 1978, and Leeds University, UK in 1996. He has published more than 100 technical paper in national and international journals, and also four textbooks. His scientific interests include optimal control, optimization, sliding mode control, bio-mathematics, ODE's, PDE's, and approximation techniques.

How to cite this paper: Ali Ketabdari, Mohammad Hadi Farahi, "A Novel Method to Solve a Class of Non-local Diffusion Optimal Control Problems by using Bernstein Polynomials", International Journal of Intelligent Systems and Applications(IJISA), Vol.12, No.3, pp.35-45, 2020. DOI: 10.5815/ijisa.2020.03.05 